BMJ Open

Diabetes

Research

\& Care

\section{Association of fasting plasma glucose variability with gestational diabetes mellitus: a nationwide population- based cohort study}

To cite: Kim JA, Kim J, Roh E, et al. Association of fasting plasma glucose variability with gestational diabetes mellitus: a nationwide population-based cohort study. BMJ Open Diab Res Care 2020;8:e001084. doi:10.1136/ bmjdrc-2019-001084

- Additional material is published online only. To view please visit the journal online (http://dx.doi.org/10.1136/ bmjdrc-2019-001084).

Received 28 November 2019 Revised 26 February 2020 Accepted 24 March 2020

Check for updates

(C) Author(s) (or their employer(s)) 2020. Re-use permitted under CC BY-NC. No commercial re-use. See rights and permissions. Published by BMJ.

${ }^{1}$ Division of Endocrinology and Metabolism, Department of Internal Medicine, College of Medicine, Korea University, Seoul, Republic of Korea ${ }^{2}$ Smart Healthcare Cancer, Korea University Guro Hospital, Seoul, Republic of Korea ${ }^{3}$ Department of Obstetrics and Gynecology, Korea University Guro Hospital, Korea University College of Medicine, Seoul, Republic of Korea

Correspondence to Dr Hye Jin Yoo; deisy21@naver.com and Dr Geum Joon Cho; md_cho@hanmail.net

\section{ABSTRACT}

Objective Long-term glycemic variability has recently been recognized as another risk factor for future adverse health outcomes. We aimed to evaluate the risk of gestational diabetes mellitus (GDM) according to the prepregnancy long-term fasting plasma glucose (FPG) variability.

Research design and methods A total of 164053 women who delivered their first baby between January 1, 2012 and December 31, 2015, were selected from the Korean National Health Insurance data. All women underwent at least three national health screening examinations, and the last examination should be conducted within 2 years before their first delivery. GDM was defined as the presence of more than four times of claim of GDM (International Classification of Disease, 10th Revision (ICD-10) 024.4 and 024.9) or prescription of insulin under the ICD-code of GDM. FPG variability was assessed by variability independent of the mean (FPGVIM), coefficient of variation, SD, and average successive variability.

Results Among the 164053 women, GDM developed in 6627 (4.04\%). Those in the higher quartiles of FPG-VIM showed a stepwise increased risk of GDM. In fully adjusted model, the ORs for GDM was 1.22 (95\% Cl 1.14 to 1.31) in women with the highest FPG-VIM quartile compared with those in the lowest quartile. The risk for GDM requiring insulin therapy was $48 \%$ increase in women in the highest quartile of FPG-VIM compared with those in the lowest quartile, while that for GDM not requiring insulin therapy was 19\% increase. The association between high FPG variability and the risk of GDM was intensified in the obese and aged more than 35 years women.

Conclusions Increased FPG variability in the prepregnancy state is associated with the risk of GDM independent of confounding factors. Therefore, prepregnancy FPG variability might be a surrogate marker of the risk of GDM.

\section{INTRODUCTION}

Parallel with the increasing prevalence of diabetes, obesity, and the late age of pregnancy, gestational diabetes mellitus (GDM) has become one of the most prevalent

\section{Significance of this study}

What is already known about this subject?

- Long-term glycemic variability has recently been recognized as a risk factor for future adverse health outcomes.

- Although gestational diabetes mellitus (GDM) affects the mother and their offspring during the perinatal period and the life-long period, it is hard to find out GDM in early stage in primipara.

What are the new findings?

- Among the 164053 women, GDM developed in 6627 $(4.04 \%)$ and women in the higher quartiles of fasting plasma glucose (FPG)-variability independent of the mean (VIM) showed a stepwise increased risk of GDM.

- The risk for GDM was $22 \%$ in the highest quartile of FPG-VIM compared with those in the lowest quartile after adjusting risk factors.

- The association between high FPG variability and the risk of GDM was intensified in the obese and aged more than 35 years.

How might these results change the focus of research or clinical practice?

- The early risk stratification of GDM using prepregnancy FPG variability might be clinically valuable, especially for the high-risk women such as obese, aged women.

pregnancy-related complications. ${ }^{1}$ GDM is associated with pre-eclampsia, preterm delivery, fetal overgrowth, neonatal hypoglycemia, and increased mortality in the perinatal period. ${ }^{2}$ Furthermore, women with previous GDM and offspring exposed to maternal hyperglycemia have an increased risk of obesity, type 2 diabetes (T2DM), and cardiometabolic diseases. ${ }^{3-5}$ As a result, GDM causes a continuous intergenerational transfer of metabolic disturbances; thus, early identification of women at risk for GDM and 
intensive strategies for reducing risk factors is critical to break this vicious cycle. However, there has been considerable controversy regarding the early screening method for GDM.

Recent growing evidences have emphasized the role of variability of certain metabolic parameters in predicting the risk for various adverse health outcomes ${ }^{6-8}$ Representatively, Kim $e t a l^{7}$ found a graded association between the number of high-variability parameters including fasting plasma glucose (FPG) and total cholesterol (TC) concentrations, systolic blood pressure (BP), and body mass index (BMI) and primary outcomes such as mortality and cardiovascular events. Long-term glycemic variability, usually demonstrated as a visit-to-visit FPG or HbA1C variability, indicates glucose fluctuation over months to years. ${ }^{9}$ The excess glycemic excursion associated with increased oxidative stress, endothelial dysfunction, and subclinical inflammation promotes organ damage. ${ }^{10}$ In human umbilical vein endothelial cells, glucose oscillation induces more oxidative stress than consistent hyperglycemia, resulting in the acceleration of apoptosis and inflammatory reactions. ${ }^{11}$ Although previous studies have extensively investigated the intimate relationship between glycemic variability and micro/macrovascular complications and overall mortality in patients with diabetes, ${ }^{12} 13$ only a few have examined the prognostic value of long-term glycemic variability in individuals without diabetes. Recently, Wang et $a l^{14}$ showed that FPG variability over 4 years was an independent predictor of cardiovascular diseases (CVD) and all-cause mortality in the general population. Bancks et $a l^{15}$ also reported that greater FPG variability during young adulthood was associated with higher risk for incident diabetes, CVD, and all-cause mortality during the middle age years. In a nationwide population study including subjects aged $\geq 40$ years without diabetes, a 1 SD increase in the FPG variability was associated with $24 \%$ increased risk of T2DM within the 8.3-year follow-up. ${ }^{16}$ However, no previous study has explored association of prepregnancy longterm FPG variability on the risk of GDM, especially to the primipara with baseline normal fasting glucose level, as GDM is difficult to detect during the early stage.

Therefore, to search for a novel risk factor for GDM, we aimed to investigate the association between prepregnancy FPG variability and the risk of GDM in the primipara using the large-scale Korean National Health Insurance Service (NHIS) database.

\section{MATERIALS AND METHODS}

\section{Data source}

The data were obtained from the NHIS database from 2002 to 2015. The NHIS is operated by the Korean government, and all enrolled participants are required to undergo annual or biannual health examinations. The NHIS database is composed of anonymous personal information, health examination database, demographics, primary and secondary diagnoses, hospitalization, and medical treatment. In this study, 1625913 women who delivered their first baby between January 1, 2012 and December 31, 2015, who underwent health examinations within 2 years before delivery (index year), and who underwent additional health examinations $(\geq 2)$ within 5 years before the last health check-up (index year) were included $(n=180094)$. We excluded women who had multiple births $(\mathrm{n}=27266)$, multiparity $(\mathrm{n}=789033)$, $\mathrm{FPG} \geq 100 \mathrm{mg} / \mathrm{dL}$ at index year $(\mathrm{n}=15582)$, a history of diabetes before index year $(\mathrm{n}=19)$, and had at least one missing data in BMI, hemoglobin, weight, BP, smoking, drinking, and exercise $(\mathrm{n}=440)$ (figure 1). In total, 164053 women were eligible for the analysis. Women with GDM were identified using the International Classification of Disease, 10th Revision (ICD-10) (O24.4 and O24.9). Categorically, 157 426, 5808, and 819 women were subgrouped as follows: normal pregnancy, GDM without insulin therapy, and GDM with insulin therapy. Our study protocol was approved by the Korea University institutional review board (IRB) and was conducted in accordance with the Declaration of Helsinki of the World Medical Association (2019GR0106). An informed consent exemption was granted by the IRB since, for the purposes of this study, only anonymous and deidentified information were provided by the NHIS.

\section{Assessment of FPG variability indices}

FPG variability was calculated using FPG values of serial health examinations. Variability independent of the mean (FPG-VIM) was defined as $100 \times \mathrm{SD} / \mathrm{mean}^{\beta}$, where $\beta$ is the regression coefficient, on the basis of the natural $\log (\ln )$ of the SD over the ln of the mean. Besides FPV-VIM, we used a coefficient of variation (FPG-CV), FPG-SD, and average successive variability (FPG-ASV); defined as the average absolute difference between successive values, to evaluate the variability of FPG. The detailed description of the calculation of various FPG variability indices has been reported in previous publication. ${ }^{17}$

\section{Definitions}

GDM was defined as the presence of more than four times of claim of GDM (ICD-10 O24.4 and O24.9) or prescription of insulin under the ICD-code of GDM. In South Korea, the "two-step" approach with a $50 \mathrm{~g}$ screen followed by a $100 \mathrm{~g}$ oral glucose tolerance test for those who screen positive is mainly used for the diagnosis of GDM. Pregnancy was defined as a day before 280 days of delivery. Diabetes was defined using the ICD-10 codes E10-14 or as the level of fasting glucose of $\geq 126 \mathrm{mg}$ / dL during the national health examinations. Heavy alcohol consumption was defined as drinking more than three times a week, and regular exercise was defined as moderate-intensity physical activity for more than $30 \mathrm{~min}$ at least 5 days a week, and high-intensity physical activity for more than $20 \mathrm{~min}$ at least 3 days a week. BMI was defined as body weight $(\mathrm{kg})$ divided by height ${ }^{2}\left(\mathrm{~m}^{2}\right)$. All blood samples were drawn after more than 8 hours of fasting. 


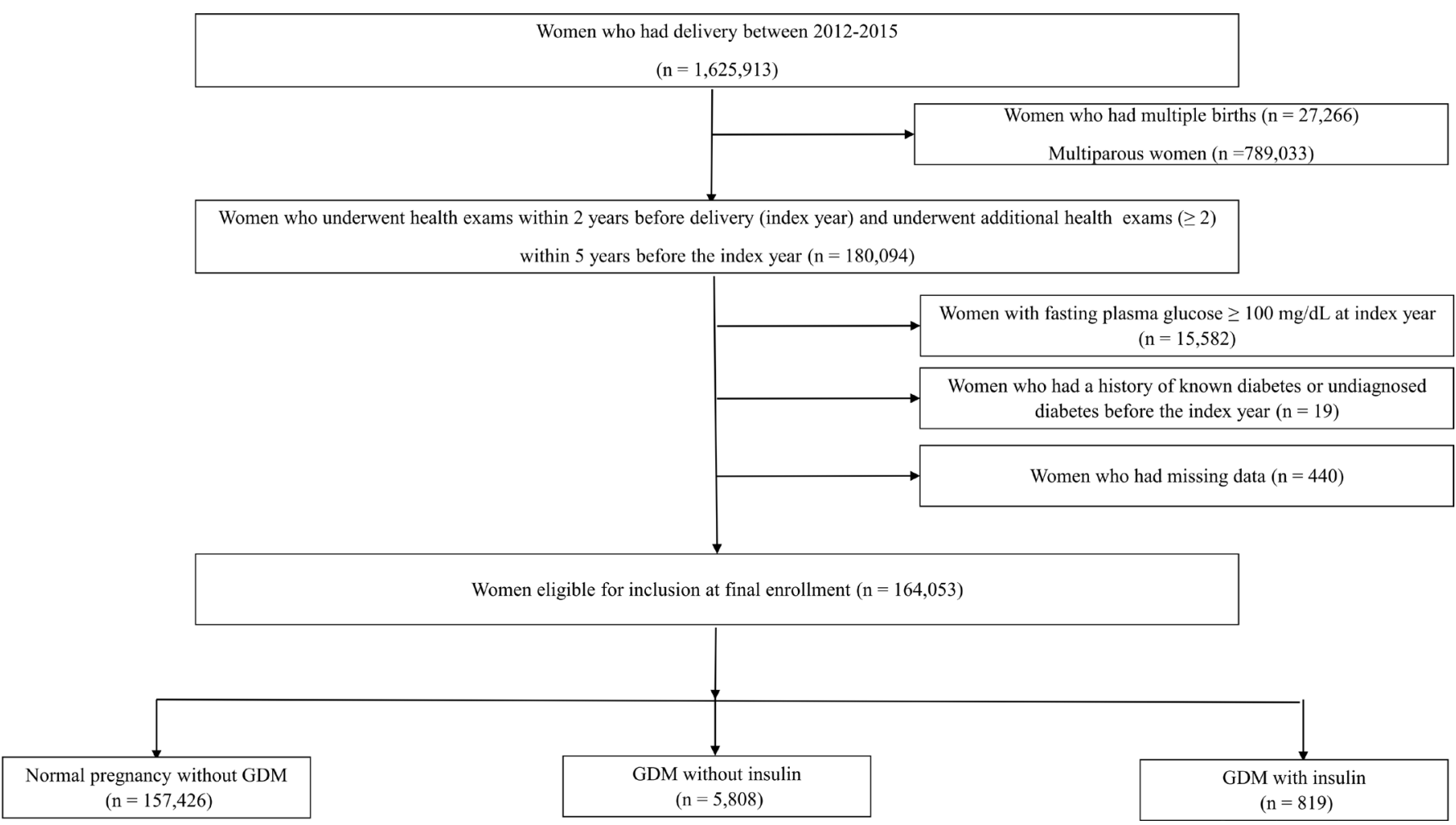

Figure 1 Flowchart of the study. GDM, gestational diabetes mellitus.

\section{Statistical analysis}

Continuous variables and categorical variables are expressed as mean $\pm \mathrm{SD}$ or as a number (percentage). To compare baseline characteristics between groups, t-test was used for continuous variables, while $\chi^{2}$ test was used for categorical variables. Subsequently, the linear trend test was performed to determine the presence of a linear trend. Multivariate logistic regression analysis was used to calculate the ORs and $95 \% \mathrm{CI}$ for the development of GDM according to the quartiles of FPG variability after adjustment for covariates including age, BMI, smoking status, heavy alcohol consumption, regular exercise, family history of diabetes, and mean FPG. The mean FPG level was defined as the average value of all fasting glucose measurements in the index year and within the previous 5 years. Sensitivity analysis was done after the exclusion of women with impaired FPG $(100 \mathrm{mg} / \mathrm{dL}<\mathrm{FPG} \leq 126 \mathrm{mg} / \mathrm{dL})$ or diagnosed with polycystic ovarian syndrome (PCOS) during the health examination from January 2002 to pregnancy. Stratified analyses were conducted according to age ( $<35$ years or $\geq 35$ years), BMI ( $<25 \mathrm{~kg} / \mathrm{m}^{2}$ or $\left.\geq 25 \mathrm{~kg} / \mathrm{m}^{2}\right)$, smoking status, heavy alcohol consumption, exercise, and family history of diabetes using the likelihood ratio test. Statistical analyses were performed using SAS V.9.4 (SAS Institute, Cary, North Carolina, USA), and $\mathrm{p}<0.05$ were considered significant.

\section{RESULTS}

Baseline characteristics

Among the 164053 primipara with normal fasting glucose level, GDM developed in 6627 (4.04\%) women who received insulin treatment $(0.50 \%)$ and who did not receive insulin treatment $(3.54 \%)$ (figure 1$)$. Women with GDM had higher age, BMI, body weight, systolic BP, diastolic BP, TC, FPG, mean FPG, FPG variability, prevalence of current smoking, and family history of diabetes than those without GDM (online supplementary table 1). Table 1 shows the baseline characteristics of women according to quartiles of FPG-VIM. BMI, systolic $\mathrm{BP}$, diastolic BP, prevalence of heavy drinking, current smoker, and family history of diabetes were increased according to FPG quartile increment ( $p$ for linear trend $<0.001)$.

\section{Risk of GDM according to the quartiles of FPG-VIIM}

Table 2 shows the risk of developing GDM according to quartiles of FPG-VIM. The risk of GDM showed a stepwise increase according to the quartiles of FPG-VIM ( $p$ for linear trend $<0.001)$. The ORs and 95\% CI of GDM total were 1.10 (1.02 to 1.18 ), 1.13 (1.05 to 1.27 ), and 1.22 (1.14 to 1.31) in the second, third, and fourth quartile FPG-VIM group, respectively, compared with those in the first FPG-VIM group after adjusting for age, BMI, smoking status, alcohol consumption, exercise, family history of diabetes, and mean FPG. In particular, the risk for GDM requiring insulin therapy increased (almost 48\%) in women in the highest quartile of FPG-VIM compared with those in the lowest quartile of FPG-VIM. Moreover, there was a modest increase $(19 \%)$ in the risk for GDM without requiring insulin therapy in the highest FPG-VIM group. Similar OR values and trends were observed in the other indices of FPG variability assessed by $\mathrm{CV}, \mathrm{SD}$, and ASV (online supplementary table 2). 
Table 1 Baseline characteristics of subjects according to quartiles of fasting glucose variability assessed by VIM (FPG-VIM)

\begin{tabular}{|c|c|c|c|c|c|c|}
\hline \multirow[b]{3}{*}{$\mathbf{N}$} & \multicolumn{4}{|l|}{ Total } & \multirow[b]{3}{*}{$P$ value } & \multirow[b]{3}{*}{ P for trend } \\
\hline & Q1 & Q2 & Q3 & Q4 & & \\
\hline & 41015 & 41011 & 41012 & 41015 & & \\
\hline Age (years) & $30.82 \pm 3.14$ & $30.77 \pm 3.10$ & $30.73 \pm 3.08$ & $30.66 \pm 3.08$ & $<0.001$ & $<0.001$ \\
\hline Body mass index $\left(\mathrm{kg} / \mathrm{m}^{2}\right)$ & $20.55 \pm 2.55$ & $20.62 \pm 2.60$ & $20.66 \pm 2.64$ & $20.71 \pm 2.69$ & $<0.001$ & $<0.001$ \\
\hline Body weight (kg) & $53.68 \pm 7.24$ & $53.83 \pm 7.38$ & $53.90 \pm 7.52$ & $53.88 \pm 7.62$ & $<0.001$ & $<0.001$ \\
\hline Systolic blood pressure (mm Hg) & $109.18 \pm 10.48$ & $109.52 \pm 10.56$ & $109.71 \pm 10.47$ & $110.22 \pm 10.54$ & $<0.001$ & $<0.001$ \\
\hline Diastolic blood pressure $(\mathrm{mm} \mathrm{Hg})$ & $68.67 \pm 7.88$ & $68.96 \pm 7.96$ & $69.11 \pm 7.89$ & $69.48 \pm 7.95$ & $<0.001$ & $<0.001$ \\
\hline Total cholesterol (mg/dL) & $176.83 \pm 28.03$ & $176.94 \pm 28.47$ & $176.96 \pm 28.70$ & $176.72 \pm 29.05$ & 0.625 & 0.631 \\
\hline Fasting glucose (mg/dL) & $86.05 \pm 6.05$ & $85.03 \pm 6.64$ & $85.77 \pm 7.41$ & $84.62 \pm 9.20$ & $<0.001$ & $<0.001$ \\
\hline Mean FPG (mg/dL) & $85.96 \pm 2.68$ & $85.87 \pm 5.59$ & $85.67 \pm 5.59$ & $85.67 \pm 7.10$ & $<0.001$ & $<0.001$ \\
\hline FPG variability (VIM) & $2.85 \pm 0.96$ & $5.25 \pm 0.59$ & $7.48 \pm 0.74$ & $12.32 \pm 4.05$ & $<0.001$ & $<0.001$ \\
\hline Alcohol consumption (\%) & $1032(2.52)$ & $1127(2.75)$ & 1266 (3.09) & $1334(3.25)$ & $<0.001$ & $<0.001$ \\
\hline Current smoker (\%) & $2074(5.06)$ & $2244(5.47)$ & 2344 (5.72) & $2491(6.07)$ & $<0.001$ & $<0.001$ \\
\hline Regular exercise (\%) & $744(1.81)$ & $749(1.83)$ & $803(1.96)$ & $829(2.02)$ & 0.080 & 0.013 \\
\hline Family history of diabetes (\%) & 30607 (74.62) & 31799 (77.54) & 32698 (79.73) & $33118(80.75)$ & $<0.001$ & $<0.001$ \\
\hline
\end{tabular}

Data are expressed as the means \pm SD or $\mathrm{n}(\%)$.

FPG, fasting plasma glucose; VIM, variability independent of the mean.

\section{Risk of GDM according to the deciles of FPG-VIIM}

When the total participants were divided into subgroups according to deciles of FPG-VIM, there were significant linear associations between deciles of FPG-VIM and the OR of GDM ( $p$ for linear trend $<0.001$ ) (figure 2 or online supplementary table 3 ). In the fully adjusted model, the

\begin{tabular}{|c|c|c|c|c|c|c|}
\hline & Events (n) & Unadjusted & Model 1 & Model 2 & Model 3 & Model 4 \\
\hline \multicolumn{7}{|l|}{ GDM total } \\
\hline Q1 (n=41015) & 1484 & 1 & 1 & 1 & 1 & 1 \\
\hline Q2 $(n=41011)$ & 1621 & 1.10 (1.02 to 1.18$)$ & 1.10 (1.03 to 1.19$)$ & 1.10 (1.02 to 1.18$)$ & 1.10 (1.02 to 1.18$)$ & 1.10 (1.02 to 1.18$)$ \\
\hline Q3 $(n=41012)$ & 1656 & 1.12 (1.04 to 1.20$)$ & 1.14 (1.06 to 1.22$)$ & 1.12 (1.04 to 1.21$)$ & 1.12 (1.04 to 1.20$)$ & 1.13 (1.05 to 1.27$)$ \\
\hline Q4 (n=41015) & 1866 & 1.27 (1.18 to 1.36$)$ & 1.30 (1.21 to 1.39$)$ & 1.27 (1.19 to 1.37$)$ & 1.27 (1.18 to 1.36$)$ & $1.22(1.14$ to 1.31$)$ \\
\hline$P$ value & & $<0.001$ & $<0.001$ & $<0.001$ & $<0.001$ & $<0.001$ \\
\hline$P$ for linear trend & & $<0.001$ & $<0.001$ & $<0.001$ & $<0.001$ & $<0.001$ \\
\hline \multicolumn{7}{|c|}{ GDM without insulin } \\
\hline Q1 $(n=40854)$ & 1323 & 1 & 1 & 1 & 1 & 1 \\
\hline Q2 (n=40 826) & 1436 & 1.09 (1.01 to 1.18$)$ & $1.10(1.02$ to 1.18$)$ & 1.09 (1.01 to 1.18$)$ & 1.09 (1.01 to 1.18$)$ & 1.09 (1.01 to 1.18$)$ \\
\hline Q3 $(n=40815)$ & 1459 & 1.11 (1.03 to 1.20$)$ & $1.12(1.04$ to 1.21$)$ & 1.11 (1.03 to 1.20$)$ & $1.11(1.03$ to 1.20$)$ & 1.12 (1.04 to 1.21$)$ \\
\hline Q4 (n=40739) & 1590 & 1.21 (1.13 to 1.31$)$ & 1.24 (1.15 to 1.33$)$ & 1.22 (1.13 to 1.32$)$ & 1.22 (1.13 to 1.32$)$ & 1.19 (1.10 to 1.28$)$ \\
\hline$P$ value & & $<0.001$ & $<0.001$ & $<0.001$ & $<0.001$ & $<0.001$ \\
\hline$P$ for linear trend & & $<0.001$ & $<0.001$ & $<0.001$ & $<0.001$ & $<0.001$ \\
\hline \multicolumn{7}{|l|}{ GDM with insulin } \\
\hline Q1 (n=39692) & 161 & 1 & 1 & 1 & 1 & 1 \\
\hline Q2 (n=39575) & 185 & 1.15 (0.93 to 1.43$)$ & 1.17 (0.94 to 1.44$)$ & $1.14(0.92$ to 1.41$)$ & $1.13(0.91$ to 1.39$)$ & $1.13(0.92$ to 1.40$)$ \\
\hline Q3 (n=39553) & 197 & 1.23 (1.00 to 1.51$)$ & 1.25 (1.02 to 1.54$)$ & 1.21 (0.98 to 1.49$)$ & 1.18 (0.96 to 1.46$)$ & 1.20 (0.98 to 1.49$)$ \\
\hline Q4 (n=39425) & 276 & 1.73 (1.43 to 2.10$)$ & 1.78 (1.47 to 2.17$)$ & 1.70 (1.39 to 2.06$)$ & 1.66 (1.36 to 2.01$)$ & $1.48(1.21$ to 1.80$)$ \\
\hline$P$ value & & $<0.001$ & $<0.001$ & $<0.001$ & $<0.001$ & $<0.001$ \\
\hline$P$ for linear trend & & $<0.001$ & $<0.001$ & $<0.001$ & $<0.001$ & $<0.001$ \\
\hline
\end{tabular}

Model 1: adjusted for age.

Model 2: adjusted for model 1 plus body mass index.

Model 3: adjusted for model 2 plus smoking status, alcohol consumption, regular exercise, family history of diabetes.

Model 4: adjusted for model 3 plus mean fasting plasma glucose.

FPG, fasting plasma glucose; GDM, gestational diabetes mellitus; VIM, variability independent of the mean. 

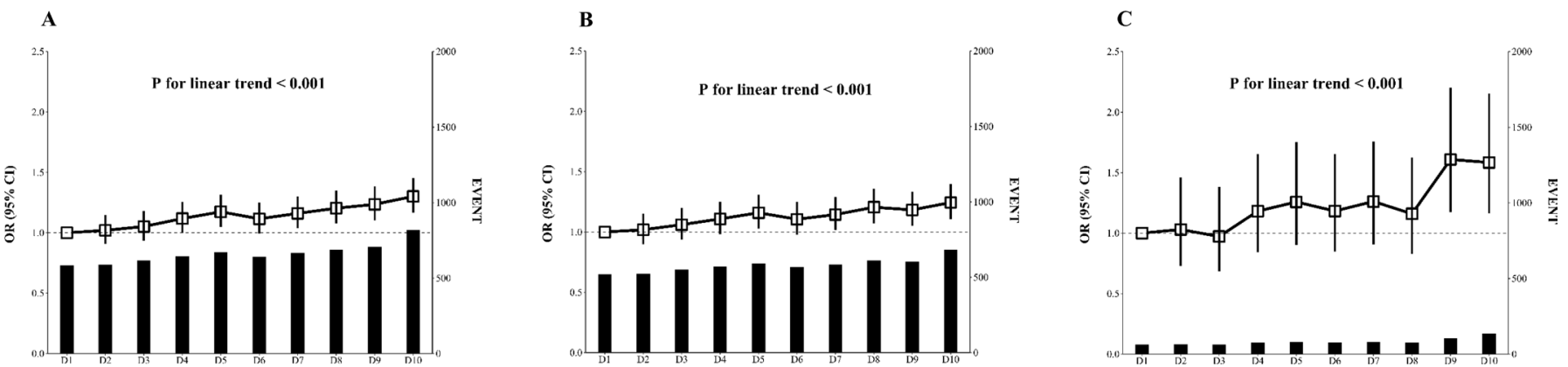

Figure 2 ORs $(95 \% \mathrm{Cl})$ of newly diagnosed GDM by deciles of FPG-VIM. (A) GDM total, (B) GDM without insulin, (C) GDM with insulin. FPG, fasting plasma glucose; GDM, gestational diabetes mellitus; VIM, variability independent of the mean.

ORs for GDM total, GDM without requiring insulin treatment, and GDM requiring insulin treatment were 1.30 , 1.25 , and 1.58 in women in the highest decile of FPG-VIM compared with those in women in the lowest decile of FPG-VIM, respectively (figure 2 or online supplementary table 3).

\section{Sensitivity analysis}

The association between FPG-VIM and GDM was not attenuated after excluding women with FPG 100-126 mg/ $\mathrm{dL}$ at least once during the health examinations from January 2002 to pregnancy (online supplementary table 4) or previous history of PCOS after adjusting for confounding factors including mean FPG level (online supplementary table 5).

\section{Subgroup analysis}

We performed stratified analysis and interaction testing to assess the potential effect modification of the risk factors of GDM including age, BMI, smoking, heavy drinking, regular exercise, and family history of diabetes (figure 3). When we compared the group in the highest quartile of FPG-VIM with those in the lowest quartile of FPG-VIM, the effect of the FPG-VIM was more obvious in the obese group than normal-weight group ( $p$ for interaction $<0.001)$. In the aspect of age, the trend of more increased $\mathrm{OR}$ was noted in women aged $\geq 35$ years than $<35$ years ( $\mathrm{p}$ for interaction $=0.055$ ). By contrast, no significant interaction was observed among smoking status, heavy alcohol consumption, exercise habit, and family history of diabetes.

\section{DISCUSSION}

Our study has a unique opportunity to reveal the impact of FPG variability in the prepregnancy period on the risk of GDM in the primipara with baseline normal fasting glucose level. Women in the highest quartile of FPG-VIM
Subgroup

Age

$<35$

$\geq 35$

BMI

$<25$

$\geq 25$

Current smoker

No

Yes

Heavy alcohol consumption

No

Yes

Regular exercise

No

Yes

Family history of diabetes

No

Yes

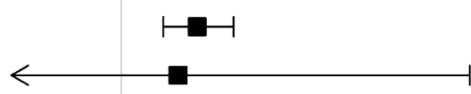

$14(1.08-1.64)$

$1.10(0.74-1.63)$

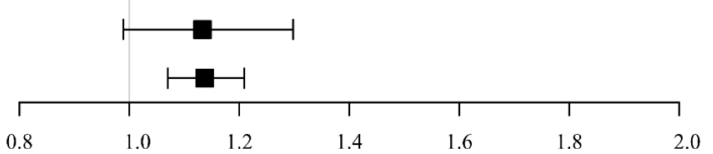

$1.13(0.99-1.30)$

$1.14(1.07-1.21)$

Figure 3 Subgroup analysis for the risk of GDM in the highest quartile versus the rest three quartiles of FPG-VIM. BMI, body mass index; FPG, fasting plasma glucose; GDM, gestational diabetes mellitus; VIM, variability independent of the mean. 
had an 22\% increased risk for GDM even after adjusting for risk factors including mean FPG, and these associations were consistent in all indices of FPG variability such as FPG-CV, FPG-SD, and FPG-ASV. In particular, there was a $48 \%$ increase in the risk for GDM requiring insulin treatment in women in the highest quartile of FPG-VIM. The association between high FPG variability and the risk of GDM was intensified in the obese and aged more than 35 years women.

The prevalence of pre-diabetes and T2DM is more than $30 \%$ in women of reproductive-age. ${ }^{18}$ However, healthy young women have less chances of being diagnosed with diabetes or impaired glucose tolerance. Although the physiologic process of GDM is complicated, ${ }^{19}$ the main pathology of GDM is an aggravation of pre-existing insulin resistance which is not compensated by insulin secretion. ${ }^{20}$ GDM causes various complications both in the mother and fetus, and the incidence of these complications can be reduced with treatment; hence, timely diagnosis is important. However, GDM is diagnosed at 24-28 weeks of gestational age using glucose loading test, which allows a very limited time to reverse the adverse outcome of hyperglycemia. A simple and cost-effective strategy for the early risk stratification of GDM is required.

Long-term glycemic variability using visit-to-visit FPG or HbA1C is a marker of glycemic homeostasis. ${ }^{9}$ Glycemic variability has been widely studied in patients with T2DM. Increased glycemic variability correlates with diabetic polyneuropathy, retinopathy, and cardiovascular autonomic neuropathy in patients with diabetes. ${ }^{12} 1321$ A metaanalysis including eight studies reported that increased FPG variability resulted in increased all-cause mortality in diabetes (HRs: 1.28, 95\% CI 1.12 to 1.46$).{ }^{13}$ Recently, the impact of glycemic variability in the general population was also highlighted. Akirov et $a t^{22}$ reported that increased glycemic variability is associated with longer hospitalization and increased short-term and long-term mortality irrespective of underlying diabetes. Furthermore, Oka et $a t^{33}$ demonstrated that higher glycemic variability was associated with more severe myocardial damage after percutaneous coronary intervention in non-diabetic patients with ST-segment elevation myocardial infarction. In the CARDIA study, adults aged 18-30 years without diabetes were followed up to 30 years, and elevated FPG variability was associated with the development of T2DM, CVD, and all-cause mortality. ${ }^{15}$ Our study is the first to demonstrate that increased FPG variability in prepregnancy was associated with the risk of GDM in the primipara with normal fasting glucose level even after adjusting other risk factors. Although FPG variability is affected by the mean glucose level, ${ }^{24}$ the association between FPG variability and GDM was consistent even after adjusting the mean FPG. Interestingly, the association of FPG variability was strengthened in insulin-treated GDM rather than in those without insulin treatment. Insulin treatment during pregnancy is the strongest risk factor for the development of T2DM; ${ }^{25}$ thus, prepregnancy FPG variability is an important risk factor for GDM and also for future glycemic derangement.

The possible pathophysiologic mechanisms connecting FPG variability and GDM could be oxidative stress and $\beta$-cell dysfunction. A previous study showed that glycemic variability measured by continuous glucose monitoring is associated with oxidative stress. ${ }^{26}$ Monnier $e t a l^{10}$ demonstrated that a marker of oxidative stress was significantly elevated in patients with high glycemic variability than in those with sustained hyperglycemia. Increased glucose excursion promotes proinflammatory cytokines by oxidative stress and contributes to the development of diabetes. ${ }^{27}$ In addition, intermittent hyperglycemia could induce mitochondrial superoxide overproduction and reduce adiponectin secretion. ${ }^{28}$ Lower adiponectin has been a well-known marker of reduced glucose sensitivity. ${ }^{29}$ Previous study demonstrated that lower adiponectin levels before pregnancy are associated with GDM. ${ }^{30}$ Besides oxidative stress, insulin secretory $\beta$-cell function is important in the pathology of GDM. Glycemic variability causes apoptosis of pancreatic $\beta$-cell, ${ }^{31}$ which may result in the deterioration of glycemic control and subsequent progression of diabetes. At 24-28 weeks gestation, glycemic variability correlates with impaired early-phase insulin secretion, which is higher in hyperglycemic women than in normoglycemic women. ${ }^{32}$ On the contrary, glycemic instability itself might be a marker of an $\beta$-cell dysfunction in at-risk individuals prior to the onset of diabetes. Women with normal glucose tolerance with a history of GDM showed elevated glycemic variability with low islet $\beta$-cell function index. ${ }^{33}$ A recent study reported that Koreans had smaller pancreatic volume and lower $\beta$-cell function than age and BMI matched Caucasians ${ }^{34}$ East Asians have limited capacity for insulin secretion. ${ }^{35}$ Hence, glycemic variability, which suggests impaired insulin secretion, can be a more useful indicator for GDM in Asian women than in women belonging to another ethnic group. Furthermore, in our study, the association between high FPG variability and the risk of GDM was strengthened in the obese women and those aged more than 35 years, which are the well-known risk factors for GDM. Therefore, the early risk stratification of GDM using prepregnancy FPG variability might be clinically valuable, especially among obese, older, and Asian women.

Our study has several limitations. First, this study is a retrospective case-control study; we could not find any causal relationship. Second, GDM women were defined as those classified using four or more GDM codes or have taken have taken insulin during pregnancy because the nationwide database had no data on $\mathrm{HbA} 1 \mathrm{C}$ value and oral glucose tolerance test results. However, the prevalence of GDM according to our definition was $4.2 \%$, which correspond to that reported in previous study. ${ }^{36}$ Finally, we did not consider dietary habits. Despite these limitations, our study has some strengths. It is the first to reveal the association between prepregnancy FPG variability and risk of GDM using large population-based 
data. In addition, to overcome the effect of the high mean glucose level in the risk of GDM, we adjusted the mean FPG level in full adjustment models.

Taken together, high glycemic variability was related to an increased risk of GDM, and the relation remained significant after the adjustment for other risk factors. GDM affects the mother and their offspring during the perinatal period and also the life-long period; however, there are no definite ways to regress GDM after diagnosis. Therefore, monitoring the longitudinal pattern of FPG level in women of reproductive age may be clinically important for the early detection of individuals with high risk for GDM. Further studies to examine the predictive power of FPG variability for the incidence of GDM should be conducted to validate these results.

Acknowledgements This work was performed in cooperation with the NHIS. The National Health Information Database constructed by the NHIS was used, and the results do not necessarily represent the opinion of the National Health Insurance Corporation.

Contributors JAK, SHB, KMC, GJC, and HJY conceived and designed the study and performed the analyses. JK, EN, and SYH conducted the statistical analysis. JK, EN, GJC, HJY, and SYH acquired the data. JAK, ER, SH, YBL, and HJY wrote the first draft of the manuscript. All authors interpreted the data, contributed to the writing of the manuscript, and read and approved the final version. GJC and HJY are responsible for the integrity of the work as a whole.

Funding This research was supported by the Basic Science Research Program through the National Research Foundation of Korea (NRF) funded by the Ministry of Education (2018R1D1A1B07047587) and the grant of the Establish R\&D Platform Project through the Korea University Medical Center and Korea University Guro Hospital, funded by the Korea University Guro Hospital (Grant number: 01903761).

Disclaimer The funders had no role in the study design, data collection, analysis, and interpretation, decision to publish, or preparation of the manuscript.

Competing interests None declared.

Patient consent for publication Not required

Ethics approval The study was approved by the Korea University institutional review board (2019GR0106).

Provenance and peer review Not commissioned; externally peer reviewed.

Data availability statement All data relevant to the study are included in the article or uploaded as supplementary information. Additional data are available on reasonable request.

Open access This is an open access article distributed in accordance with the Creative Commons Attribution Non Commercial (CC BY-NC 4.0) license, which permits others to distribute, remix, adapt, build upon this work non-commercially, and license their derivative works on different terms, provided the original work is properly cited, appropriate credit is given, any changes made indicated, and the use is non-commercial. See: http://creativecommons.org/licenses/by-nc/4.0/.

ORCID iDs

Kyung Mook Choi http://orcid.org/0000-0001-6175-0225

Hye Jin Yoo http://orcid.org/0000-0003-0600-0266

\section{REFERENCES}

1 Zhu Y, Zhang C. Prevalence of gestational diabetes and risk of progression to type 2 diabetes: a global perspective. Curr Diab Rep 2016;16:7.

2 Geurtsen ML, van Soest EEL, Voerman E, et al. High maternal earlypregnancy blood glucose levels are associated with altered fetal growth and increased risk of adverse birth outcomes. Diabetologia 2019;62:1880-90.

3 Bellamy L, Casas J-P, Hingorani AD, et al. Type 2 diabetes mellitus after gestational diabetes: a systematic review and meta-analysis. Lancet 2009;373:1773-9.

4 Clausen TD, Mathiesen ER, Hansen T, et al. High prevalence of type 2 diabetes and pre-diabetes in adult offspring of women with gestational diabetes mellitus or type 1 diabetes. The role of intrauterine hyperglycemia 2008;31:340-6.

5 Kramer CK, Campbell S, Retnakaran R. Gestational diabetes and the risk of cardiovascular disease in women: a systematic review and meta-analysis. Diabetologia 2019;62:905-14

6 Chung HS, Lee JS, Kim JA, et al. Variability in total cholesterol concentration is associated with the risk of dementia: a nationwide population-based cohort study. Front Neurol 2019;10:441.

$7 \mathrm{Kim}$ MK, Han K, Park Y-M, et al. Associations of variability in blood pressure, glucose and cholesterol concentrations, and body mass index with mortality and cardiovascular outcomes in the general population. Circulation 2018;138:2627-37.

8 Mehlum MH, Liestøl K, Kjeldsen SE, et al. Blood pressure variability and risk of cardiovascular events and death in patients with hypertension and different baseline risks. Eur Heart $J$ 2018;39:2243-51.

9 Ceriello A, Monnier L, Owens D. Glycaemic variability in diabetes: clinical and therapeutic implications. Lancet Diabetes Endocrinol 2019;7:221-30.

10 Monnier L, Mas E, Ginet C, et al. Activation of oxidative stress by acute glucose fluctuations compared with sustained chronic hyperglycemia in patients with type 2 diabetes. JAMA 2006;295:1681-7.

11 Jones SC, Saunders HJ, Qi W, et al. Intermittent high glucose enhances cell growth and collagen synthesis in cultured human tubulointerstitial cells. Diabetologia 1999;42:1113-9.

12 Yang C-P, Li C-I, Liu C-S, et al. Variability of fasting plasma glucose increased risks of diabetic polyneuropathy in T2DM. Neurology 2017;88:944-51

13 Zhao Q, Zhou F, Zhang Y, et al. Fasting plasma glucose variability levels and risk of adverse outcomes among patients with type 2 diabetes: a systematic review and meta-analysis. Diabetes Res Clin Pract 2019;148:23-31.

14 Wang A, Liu X, Xu J, et al. Visit-to-Visit variability of fasting plasma glucose and the risk of cardiovascular disease and all-cause mortality in the general population. J Am Heart Assoc 2017;6. doi:10.1161/JAHA.117.006757. [Epub ahead of print: 29 Nov 2017].

15 Bancks MP, Carson AP, Lewis CE, et al. Fasting glucose variability in young adulthood and incident diabetes, cardiovascular disease and all-cause mortality. Diabetologia 2019;62:1366-74.

16 Kim JA, Lee JS, Chung HS, et al. Impact of Visit-to-Visit fasting plasma glucose variability on the development of type 2 diabetes: a nationwide population-based cohort study. Diabetes Care 2018;41:2610-6.

17 Echouffo-Tcheugui JB, Zhao S, Brock G, et al. Visit-to-Visit glycemic variability and risks of cardiovascular events and all-cause mortality: the ALLHAT study. Diabetes Care 2019;42:486-93.

18 Hod M, Kapur A, Mclntyre HD, et al. Evidence in support of the International association of diabetes in pregnancy study groups' criteria for diagnosing gestational diabetes mellitus worldwide in 2019. Am J Obstet Gynecol 2019;221:109-16.

19 Powe CE, Allard C, Battista M-C, et al. Heterogeneous contribution of insulin sensitivity and secretion defects to gestational diabetes mellitus: table 1. Diabetes Care 2016;39:1052-5.

20 Mclntyre HD, Catalano P, Zhang C, et al. Gestational diabetes mellitus. Nat Rev Dis Primers 2019;5:47.

21 Jun JE, Jin S-M, Baek J, et al. The association between glycemic variability and diabetic cardiovascular autonomic neuropathy in patients with type 2 diabetes. Cardiovasc Diabetol 2015;14:70.

22 Akirov A, Diker-Cohen T, Masri-Iraqi H, et al. High glucose variability increases mortality risk in hospitalized patients. J Clin Endocrinol Metab 2017;102:2230-41.

23 Oka S, Deyama J, Umetani K, et al. Glycemic variability is associated with myocardial damage in nondiabetic patients with ST-elevation myocardial infarction. Cardiovasc Endocrinol Metab 2018;7:47-53.

24 Chakarova N, Dimova R, Grozeva G, et al. Assessment of glucose variability in subjects with prediabetes. Diabetes Res Clin Pract 2019;151:56-64.

25 Lee AJ, Hiscock RJ, Wein P, et al. Gestational diabetes mellitus: clinical predictors and long-term risk of developing type 2 diabetes. A retrospective cohort study using survival analysis 2007;30:878-83.

26 Ohara M, Fukui T, Ouchi M, et al. Relationship between daily and day-to-day glycemic variability and increased oxidative stress in type 2 diabetes. Diabetes Res Clin Pract 2016;122:62-70.

27 Esposito K, Nappo F, Marfella R, et al. Inflammatory cytokine concentrations are acutely increased by hyperglycemia in humans: role of oxidative stress. Circulation 2002;106:2067-72.

28 Tsai C-J, Hsieh C-J, Tung S-C, et al. Acute blood glucose fluctuations can decrease blood glutathione and adiponectin levels in patients with type 2 diabetes. Diabetes Res Clin Pract 2012:98:257-63. 
29 Kim C. Gestational diabetes mellitus in Korean women: similarities and differences from other racial/ethnic groups. Diabetes Metab J 2014;38:1-12.

30 Hedderson MM, Darbinian J, Havel PJ, et al. Low prepregnancy adiponectin concentrations are associated with a marked increase in risk for development of gestational diabetes mellitus. Diabetes Care 2013;36:3930-7.

31 Del Guerra S, Grupillo M, Masini M, et al. Gliclazide protects human islet beta-cells from apoptosis induced by intermittent high glucose. Diabetes Metab Res Rev 2007;23:234-8.

32 Su J-bin, Wang X-qin, Chen J-feng, et al. Glycemic variability in gestational diabetes mellitus and its association with $\beta$ cell function. Endocrine 2013;43:370-5.
33 Wang Y-M, Zhao L-H, Su J-B, et al. Glycemic variability in normal glucose tolerance women with the previous gestational diabetes mellitus. Diabetol Metab Syndr 2015;7:82.

34 Roh E, Kim KM, Park KS, et al. Comparison of pancreatic volume and fat amount linked with glucose homeostasis between healthy Caucasians and Koreans. Diabetes Obes Metab 2018;20:2642-52.

35 Kodama K, Tojjar D, Yamada S, et al. Ethnic differences in the relationship between insulin sensitivity and insulin response: a systematic review and meta-analysis. Diabetes Care 2013;36:1789-96.

36 Jang HC. Gestational diabetes in Korea: incidence and risk factors of diabetes in women with previous gestational diabetes. Diabetes Metab J 2011;35:1-7. 